\title{
Developing a Decision Support Testing Algorithm to Detect Severity Level of Dengue
}

\author{
S.D. Pavithra Jayasundara, BSc \\ S.S.N. Perera, PhD
}

Research and Development Center for Mathematical Modelling, Faculty of

Science, University of Colombo, Sri Lanka

N. S. Rathnayaka, MPhil

Department of Mathematics, Faculty of Engineering, General Sir John

Kotelawala Defence University, Sri Lanka

doi: 10.19044/esj.2017.v13n9p137 $\quad$ URL:http://dx.doi.org/10.19044/esj.2017.v13n9p137

\begin{abstract}
Dengue is a vector borne disease that has become a global threat. In order to reduce the mortality rate early detection of dengue severity level is crucial. This study is an extension of the decision models developed individually for inflammatory mediators and immune parameters. The objective of this study is to improve the individual models by considering their combined effect and to improve the decision making at 96 hours from onset of illness. In order to combine these, three approaches are attempted including, combining together the individual full models on inflammatory mediators and immune parameters, combining the immune parameters based model with decision tree informed cytokines and implementing a decision tree informed model with immune parameters and inflammatory mediators. The decision tree algorithm that is used in model development is Improved ID3 algorithm. The decision tree based model is a two-step decision system with the initial decision being made using the parameters TNF- $\alpha$, IL-10, dengue NS1 antigen and dengue IgG antibody and, the operator values above 0.4413 , are then subjected to the second test including platelet and Platelet Activating Factor. The decision tree based model performed well with an accuracy of $76.19 \%$ and $82.3 \%$ of DHF patients were correctly classified. Sensitivity analysis indicated the model to be robust.
\end{abstract}

Keywords: Dengue, Decision tree, Fuzzy Logic, Immune parameters, cytokines 


\section{Introduction}

Dengue is one of the most concerned mosquito borne viral diseases in the world with the reported dengue cases increasing worldwide from 2.2 million in 2010 to 3.2 million in 2015 (World Health Organization, 2016). In 2013, estimated 9000 deaths occurred globally with a majority being reported from low and middle income countries (Stanaway, et al., 2016). Dengue virus which is transmitted by Aedes Aegypti and Aedes Albopictus mosquitoes can result in asymptomatic infection, dengue fever (DF) or severe forms of dengue haemorrhagic fever (DHF) and life threatening dengue shock syndrome (DSS). Although a commercial vaccine against dengue is being licensed in several countries, it is still not in use in many parts of the world which suffer from consistent dengue outbreaks (World Health Organization, 2016). Therefore, early detection and careful body fluid management remains important in treating against dengue so as to prevent a patient from moving into critical phase.

Cytokines are believed to be associated with increased vascular permeability that may lead to severe forms of dengue (Appanna, et al., 2012). In this analysis Platelet Activating Factor (PAF), sphingosine 1phosphate (S1P), Tumor Necrosis Factor $-\alpha$ (TNF- $\alpha)$ and Interleukin -10 (IL10) are used as parameters. Previous studies have shown the impact that cytokines and inflammatory mediators can have on determining dengue disease severity. Elevated levels are observed for IL-1 $\beta$, IFN- $\gamma$, IL-4, IL-6, IL-13 and IL-7 in DHF patients than in DF patients and TNF- $\alpha$ is shown to be associated with thrombocytopenia (Bozza, et al., 2008). Also, compared with DF patients, DHF patients have shown to have significantly lower S1P levels throughout the course of illness than DF patients (Gomes, et al., 2014).

Immune parameters used in the study are dengue NS1 antigen levels, dengue IgG antibody levels, platelet counts and lymphocyte counts. NS1 positivity is found to be associated with severe dengue, especially on day 5-6 after illness. Also, it is found to be inversely correlated with lymphocyte count (Paranavitane, et al., 2014). During a secondary dengue infection IgG is present in high values even in the acute phase and thus $\operatorname{IgG/IgM}$ antibody ratios are used to distinguish between primary and secondary infection (World Health Organization, 2009). Thrombocytopenia is believed to be a resultant of bone marrow suppression and peripheral destruction of platelets (de Azeredo, et al., 2015).

This study is an extension of the studies in (Jayasundara, et al., 2017) and (Premaratne, et al., 2017) in which fuzzy based decision models are developed separately for inflammatory mediators (Jayasundara, et al., 2017) and immune parameters (Premaratne, et al., 2017). The objective of this study is to develop a decision tree informed fuzzy decision system that can detect dengue severity at an early stage. Thus, this model focuses on 
detection at 96 hours from onset of illness rather than at 108 or 120 hours from onset of illness as was the case in the previous models. This model is a combination of the previous two models and is targeted at determining disease severity at an early stage. The combined interaction of inflammatory mediators together with immune parameters is of interest to study as, previous studies have shown that S1P levels are significantly correlated with platelet counts in DHF patients (Green, et al., 1999) and IL-10 levels are significantly and inversely correlated with lymphocyte counts (Malavige, et al., 2013). Furthermore, this model targets at reducing the number of parameters that are required for decision making.

Several fuzzy logic based models are developed to detect various diseases including heart disease, diabetes, asthma and liver disorders (Anand, et al., 2013), (Kalpana \& Kumar, 2011), (Adeli \& Neshat, 2010), (Neshat, et al., 2008). Decision trees are commonly used to handle biological problems and in this study, Improved ID3 (IID3) algorithm is used to determine the effect that inflammatory mediators and immune parameters have on detecting disease severity. A classification and regression tree (CART) analysis using the parameters white blood cell count, percent monocytes, platelet count and haematocrit was designed based on a cohort of Thai children at 72 hours from onset of illness. This study achieved a $97 \%$ sensitivity in detecting patients who proceeded into DSS (Potts, et al., 2010). CART decision tree based on platelets, IL-10 and lymphocyte resulted in a model with an accuracy of $84.6 \%$ for DHF and $84.0 \%$ for DF (Brasier, et al., 2012).

\section{Development of decision support model Data}

Data was obtained from 36 adult patients who were admitted to the Colombo South Teaching Hospital, Sri Lanka. According to 2011 WHO guidelines, out of these, 11 patients are classified as DF while 25 are classified as DHF. These patients are admitted to the hospital at varying time points ranging from 72-144 hours from onset of illness. However, our analysis is limited to only 96 hours from onset of illness as the aim is for early detection and sufficient data did not exist for earlier time points. In the data set, there are $17 \mathrm{DHF}$ and 4 DF patients who are admitted at 96 hours from onset of illness.

\section{Decision Tree}

The decision tree algorithm that is used in this analysis is Improved ID3 algorithm (IID3). ID3 algorithm is a fundamental decision tree algorithm which is widely used with data having only categorical attributes. This uses information gain to determine the most suitable property for each 
node and the attribute with the highest information gain is selected as the attribute for that particular node. However, as ID3 algorithm tend to be biased towards selecting the attribute with many values, this is modified using an association function to overcome this drawback and the modified IID3 algorithm is developed (Jin, et al., 2009). The IID3 algorithm is a multy splitting algorithm that can be used with data having both catogorical and numerical attributes.

\section{Theoretical Framework}

\section{Fuzzy set}

Elements of a fuzzy set are mapped using a membership function $\mu: A \rightarrow[0,1]$ which measures the degree to which an element belongs to the set (Zadeh, 1965).

\section{Hamacher Operator}

$\mu_{\mathrm{H}}(\mathrm{x})=\left\{\begin{array}{cc}0 & \text { if } \mu_{\mathrm{A}}(\mathrm{x})=\mu_{\mathrm{B}}(\mathrm{y})=0 \\ \frac{\mu_{\mathrm{A}}(\mathrm{x})^{*} \mu_{\mathrm{B}}(\mathrm{y})}{\mu_{\mathrm{A}}(\mathrm{x})+\mu_{\mathrm{B}}(\mathrm{y})-\mu_{\mathrm{A}}(\mathrm{x})^{*} \mu_{\mathrm{B}}(\mathrm{y})} & \text { otherwise, }\end{array}\right.$

where $\mu_{A}(x), \mu_{B}(x)$ are the membership function values of the fuzzy sets $A$ and $\mathrm{B}$ respectively.

\section{Ordered Weighted Aggregation (OWA) Operator}

OWA operator is defined as,

OWA $\left(a_{1}, a_{2}, \ldots, a_{n}\right)$

$=\sum_{j=1}^{n} w_{j} b_{j}$

where $b_{j}$ is the $\mathrm{j}^{\text {th }}$ largest element of the collection of aggregated objects $a_{1}, a_{2}, \ldots, a_{n}$ and $\sum_{j=1}^{n} w_{j}=1$ and $w_{j} \in[0,1]$.

A way to determine the weights of OWA operator is suggested by (Yager, 1993) and (Yager, 1988) as,

$w_{i}=Q\left(\frac{i}{n}\right)-Q\left(\frac{i-1}{n}\right) \quad ; i=1,2, \ldots, n$

and the non-decreasing quantifier $Q$ is defined in (Zadeh, 1983) as,

$Q=\left\{\begin{aligned} 0 & ; \text { if } r<l \\ \frac{r-l}{b-m} & ; \text { if } l \leq r \leq m \\ 1 & ; \text { if } r>m\end{aligned}\right.$

where $l, m, r \in[0,1]$.

'Orness measure' which measures the degree to which the aggregation operation is like an 'OR' operation is defined by (Yager, 1988) as

$\operatorname{orness}(w)=\frac{1}{(n-1)} \sum_{i=1}^{n}(n-i) w_{i}$ 
which lies in the interval $[0,1]$.

\section{Model Development}

The fuzzy membership functions are developed according to the previous studies (Jayasundara, et al., 2017) and (Premaratne, et al., 2017).

$\mu_{10}(x)=$

$\left\{\begin{array}{cl}1 & ; x \leq 20 \\ \frac{110-x}{90} & ; 20<x<110 \\ 0 & ; x \geq 110\end{array}\right.$

$\mu_{\alpha}(x)=$

$\left\{\begin{aligned} 1 & ; x \leq 15 \\ \frac{30-x}{15} & ; 15<x<30 \\ 0 & ; x \geq 30\end{aligned}\right.$

$\mu_{P A}(x)=$

$\left\{\begin{aligned} 1 & ; x \leq 10 \\ \frac{100-x}{90} & ; 10<x<100 \\ 0 & ; x \geq 100\end{aligned}\right.$

$\mu_{S}(x)=$

$\left\{\begin{aligned} 0 & ; x \leq 0.5 \\ x-0.5 & ; 0.5<x<1.5 \\ 1 & ; x \geq 1.5\end{aligned}\right.$

The membership functions for IL-10, TNF- $\alpha$, PAF and S1P are given by the equations (3), (4), (5) and (6) respectively.

$$
\begin{aligned}
& \mu_{P}\left(x_{p}\right) \\
& =\left\{\begin{array}{cl}
0 & \text { if } x_{p} \leq 25 \\
2\left(\frac{x-25}{125}\right)^{2} & \text { if } 25<x_{p} \leq 87.5 \\
1-2\left(\frac{x-150}{125}\right)^{2} & \text { if } 87.5<x_{p} \leq 150 \\
1 & \text { if } x_{p}>150
\end{array}\right. \\
& \mu_{N}\left(x_{N}\right) \\
& =\left\{\begin{array}{cl}
1 & \text { if } x_{N}=0 \\
1-2\left(\frac{x}{75}\right)^{2} & \text { if } 0<x_{N} \leq 37.5 \\
2\left(\frac{x-75}{75}\right)^{2} & \text { if } 37.5<x_{N} \leq 75 \\
0 & \text { if } x_{N}>75
\end{array}\right.
\end{aligned}
$$




$$
\begin{aligned}
& \mu_{\mathrm{L}}\left(\mathrm{x}_{\mathrm{L}}\right) \\
& =\left\{\begin{aligned}
\frac{\mathrm{x}}{14000}-\frac{1}{140} & \text { if } 100<\mathrm{x}_{\mathrm{L}} \leq 1500 \\
0.9 * \frac{\mathrm{x}}{4500}-0.2 & \text { if } 1500<\mathrm{x}_{\mathrm{L}} \leq 6000 \\
1 & \text { if } \mathrm{x}_{\mathrm{L}}>6000
\end{aligned}\right. \\
& \mu_{\mathrm{I}}\left(\mathrm{x}_{\mathrm{I}}\right) \\
& =\left\{\begin{aligned}
\frac{-\mathrm{x}}{36}+1 & \text { if } \mathrm{x}_{\mathrm{I}}<18 \\
0.5 & \text { if } 18<\mathrm{x}_{\mathrm{I}} \leq 22 \\
\frac{-\mathrm{x}}{156}+\frac{25}{39} & \text { if } 22<\mathrm{x}_{\mathrm{I}} \leq 100 \\
0 & \text { if } \mathrm{x}_{\mathrm{I}}>100
\end{aligned}\right.
\end{aligned}
$$

The membership functions for platelet, dengue NS1 antigen, lymphocyte and dengue IgG antibody levels are given in equations (7), (8), (9) and (10) respectively.

Three approaches are attempted to combine the individual models in Jayasundara, et al. (2016) and Premaratne, et al. (2017). For future reference, the cytokines and inflammatory mediators based model of Jayasundara, et al. (2016) is defined as Model A and immune parameters based model of Premaratne, et al. (2017) is defined as Model B.

$1^{\text {st }}$ approach: Combining together the individual models, Model A and Model B.

$2^{\text {nd }}$ approach: Combining Model B with only the decision tree informed cytokines in Model A.

$3^{\text {rd }}$ approach: Decision tree informed model consisting of immune parameters and inflammatory mediators.

\section{Decision Tree Output}

IID3 algorithm based decision trees are implemented for 96 and 108 hours from onset of illness for the parameters in Model A and Model B.

96 hours from onset of illness. The decision tree for inflammatory mediators and immune parameters evaluated at 96 hours from onset of illness is shown in Fig. 1 and Fig. 2 respectively. In the figures, $y=1$ refers to DHF patients and $y=2$ refers to DF patients and ' $n$ ' refers to the number of patients classified under that particular decision making. 


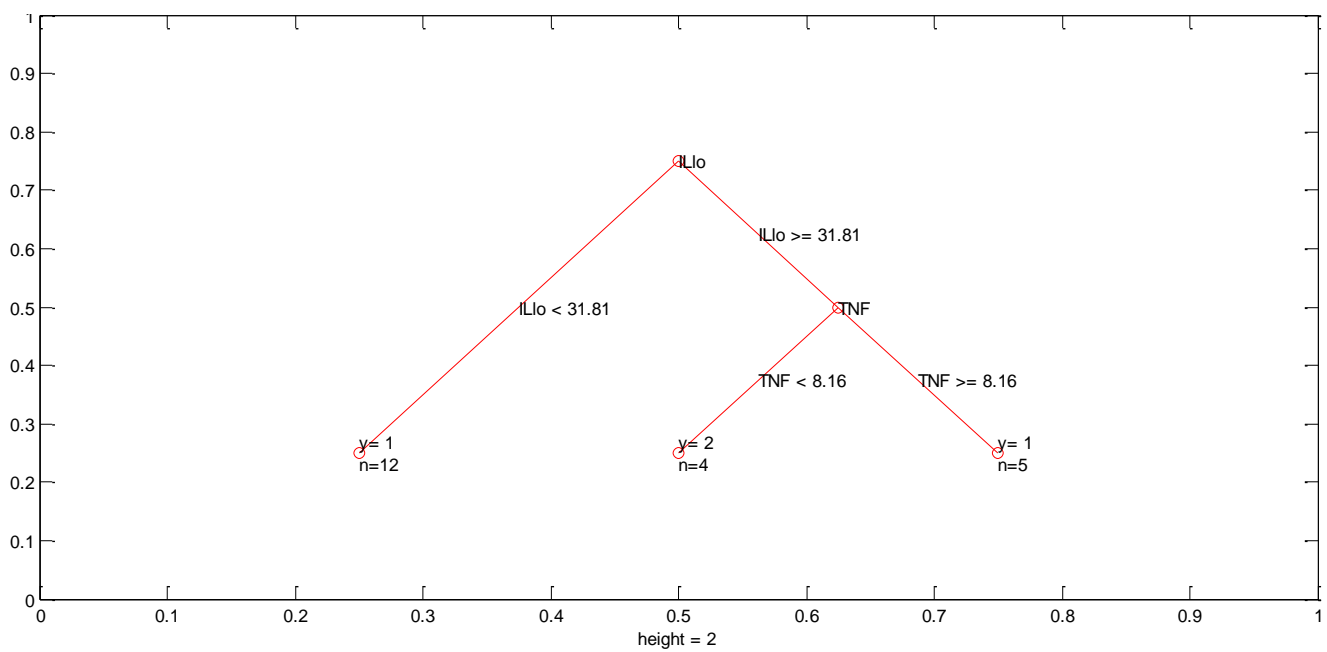

Fig.1: Decision tree at 96 hours from onset of illness for cytokines.

As it can be seen from Fig.1, at 96 hours from onset of illness decisions are made using only the parameters TNF- $\alpha$ and IL-10 and the parameters PAF and S1P are not used in the decision process. At this time point, 12 out of 17 (70.59\%) of DHF patients are classified based only on the IL-10 concentration. All four DF patients are categorized by the decision criteria IL-10 $>=31.81(\mathrm{pg} / \mathrm{ml})$ and TNF $<8.16(\mathrm{pg} / \mathrm{ml})$.

Also, according to Fig.1, if the IL-10 concentration is less than 31.81 $(\mathrm{pg} / \mathrm{ml})$ the patients are directly categorized as DHF and thus indicates that IL-10 is an important parameter in making decisions as it was in Brasier, et al. (2012). However, according to Perez, et al. (2004), Green, et al. (1999) IL-10 levels have shown to be higher in DHF patients than in DF patients and according to Chen, et al. (2006) DHF patients showed a median IL-10 level of $110.8 \mathrm{pg} / \mathrm{ml}$ and DF patients a median of $15.5 \mathrm{pg} / \mathrm{ml}$. Therefore, further analysis is required to determine if the low levels of IL-10 in DHF patients is significant or whether it is specific to this data set alone.

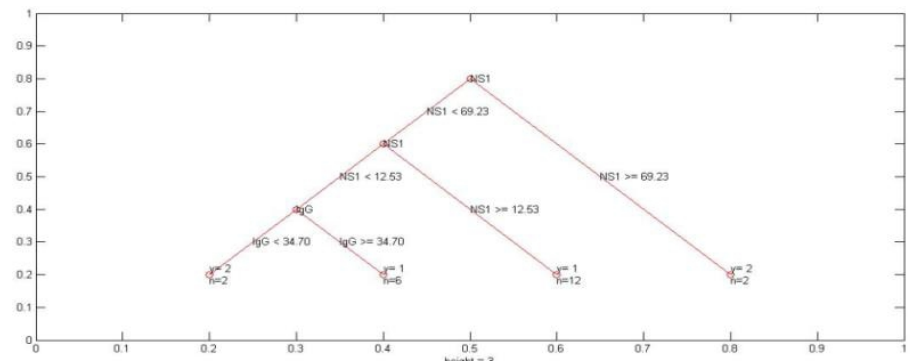

Fig.2: Decision tree at 96 hours from onset of illness for immune parameters. 
From Fig.2 it can be seen that at 96 hours from onset of illness decisions are made using only the parameters dengue NS1 antigen and dengue $\mathrm{IgG}$ antibody, indicating these parameters to be informative at 96 hours from onset of illness. The parameters platelet and lymphocyte counts are not used in the decision process.

\section{8 hours from onset of illness.}

The decision tree for inflammatory mediators and immune parameters evaluated at 108 hours from onset of illness is shown in Fig.3 and Fig. 4 respectively. In the figures, $y=1$ refers to DHF patients and $y=2$ refers to DF patients and ' $n$ ' refers to the number of patients classified under that particular decision making.

As it can be seen from Fig.3 at 108 hours from onset of illness since TNF- $\alpha$ has the highest information gain, decision making is started with TNF- $\alpha$. Decisions are made using only the parameters TNF- $\alpha$ and PAF. 13 out of $17(70.59 \%)$ of DHF patients are classified based only on TNF- $\alpha$ values.

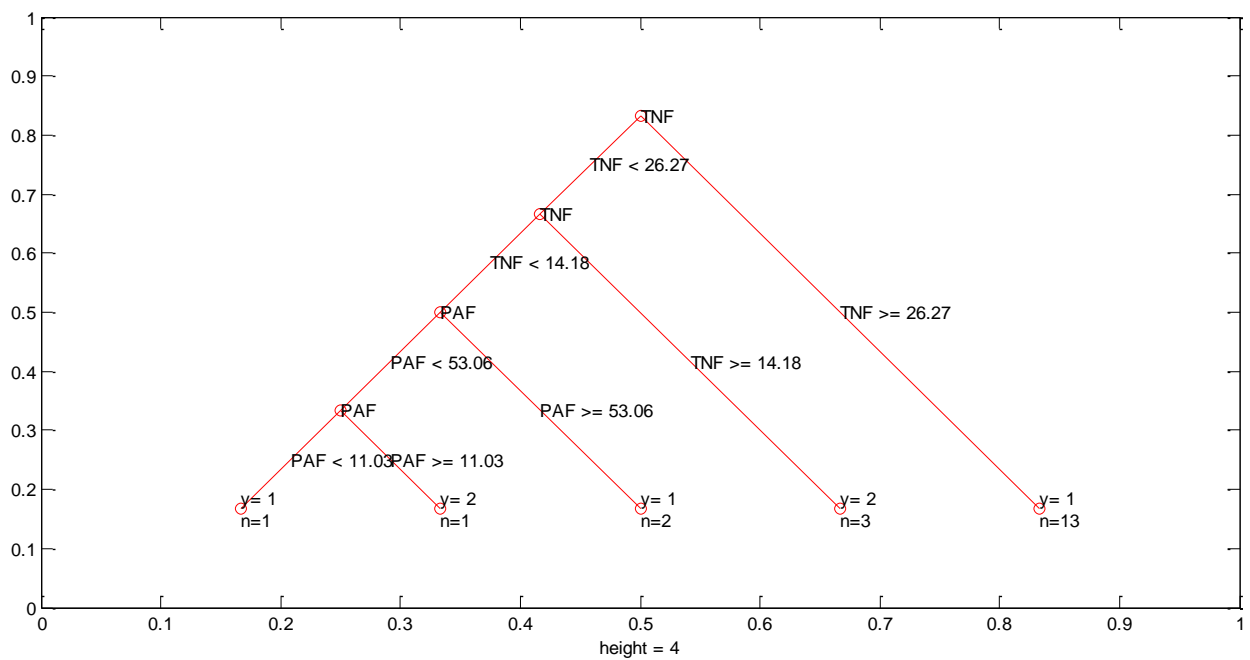

Fig.3: Decision tree at 108 hours from onset of illness for inflammatory mediators.

As it can be seen from Fig.4, at 108 hours from onset of illness since NS1 has the highest information gain, decision making is started with it. Decisions are made using only the parameters NS1, IgG and platelet count but, the lymphocyte count is not taken for consideration. 


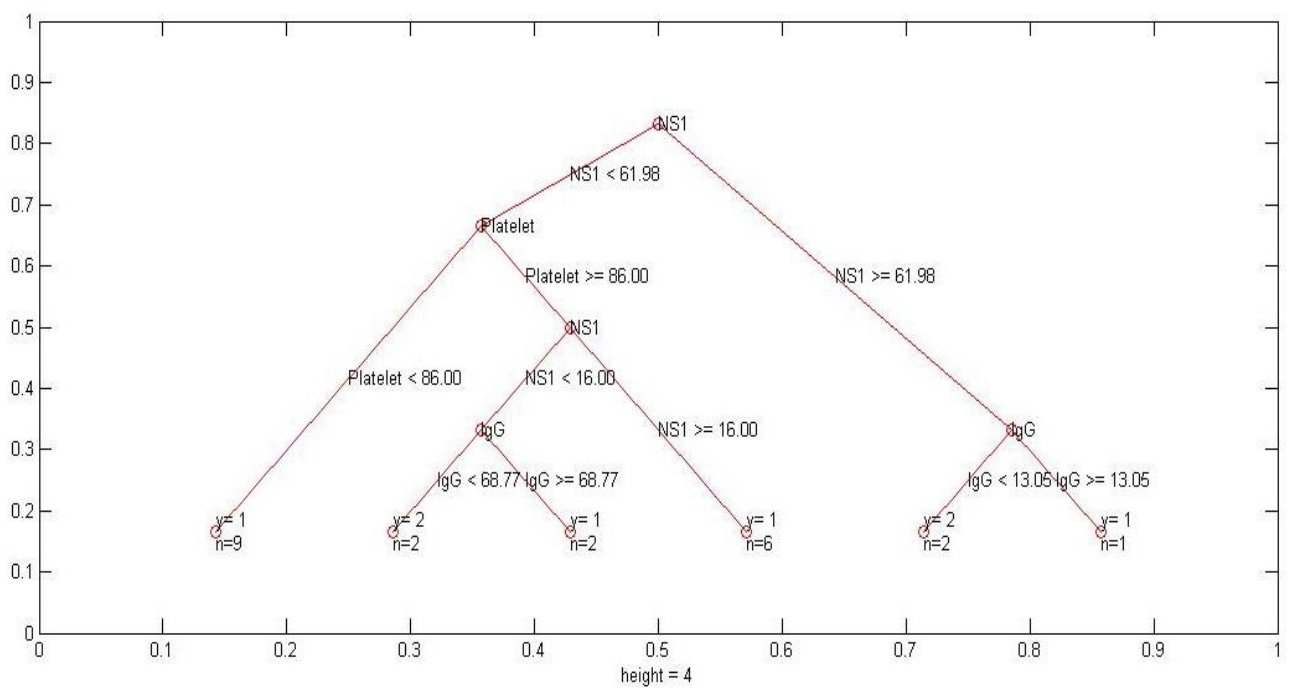

Fig.4: Decision tree at 108 hours from onset of illness for immune parameters.

\section{Results and Discussion}

$1^{\text {st }}$ approach: Combining together the individual models, Model A and Model B.

Model A and Model B are combined together by combining the individual model output values of the two models through the Hamacher operator. The accuracy of the model is calculated as,

\section{Accuracy}

$=\frac{\text { correctly classified } D H F+\text { correctly classified } D F}{\text { Total number of patients }}$

At 96 hours the combined model performed with an accuracy of $58.82 \%$. However, still $42.85 \%$ of the DHF patients' model results fell in between 0.3151-0.5264, which is the ambiguous region defined in Model B and the DF patients' results did not improve. However, none of the DHF patients fell into the non-severe region.

\section{$2^{\text {nd }}$ approach: Combining Model B with only the decision tree informed cytokines in Model A.}

Since the immune parameters in Model B are easy to measure and the inflammatory mediators are costly, it is decided to use the full Model B and improve its results by incorporating the cytokines which are indicated as informative by the decision tree. As the objective is for early detection, the decision tree results evaluated at 96 hours from onset of illness are used to modify the Model B. As it can be seen from Fig.1, since the decision tree 
results are based on the cytokines TNF- $\alpha$ and IL-10, their combined effect is considered along with the results from Model B.

In the Model B, $43.75 \%$ of the DHF patients fell into the non-severe region and $12.5 \%$ of DHF patients fell into the ambiguous region. Therefore, to improve the outcome of the misclassification in Model B, the patients whose operator value is above the lower limit of the ambiguous region (0.3151), were further subjected to the combined effect given in equation (11).

$$
\mathrm{H} 1=\frac{\mu_{\alpha}(\mathrm{x})^{1.1 *} \mu_{10}(\mathrm{x})^{1.1}}{\mu_{\alpha}(\mathrm{x})^{1.1}+\mu_{10}(\mathrm{x})^{1.1}-\mu_{\alpha}(\mathrm{x})^{1.1 *} \mu_{10}(\mathrm{x})^{1.1}}
$$

where $\mu_{\alpha}(x), \mu_{10}(x)$ are the membership values of TNF- $\alpha$ and IL-10 respectively and both are concentrated by 1.1 as in (Jayasundara, et al., 2017). H1 produces the Hamacher product between TNF- $\alpha$ and IL-10.

$\mathrm{H} 1$ is combined with the Model B results as given in equation (12).

$$
\mathrm{H} 1^{\prime}=\frac{H 1 * O W A a}{H 1+O W A a-H 1 * O W A a}
$$

where $O W A a$ refers to the final model output value given by model B.

This implementation performed with an accuracy of $64.7 \%$. However, $28.57 \%$ of the DHF patients are categorized as non-severe and the DF patients' results did not improve. The misclassification of the DHF patients into non-severe region is a concern and hence further development of the model is required.

\section{$3^{\text {rd }}$ approach: Decision tree informed model consisting of immune parameters and inflammatory mediators.}

IID3 decision tree algorithm is evaluated for 96 hours from onset of illness for the two sets of parameters, one including cytokines (Fig.1) and the other including immune parameters (Fig.2).

Since, at 96 hours from onset of illness, the decision tree based on inflammatory mediators outputs the results based only on the parameters TNF- $\alpha$ and IL-10 (Fig.1) and the immune parameters based decision tree outputs the results based only on the parameters NS1 and IgG (Fig.2), the initial decision model is developed using only these four parameters. In order to account for the combined effect from the parameters, the Hamacher operator is performed as described in equations (11) and (13). The fuzzy concentration and dilution of the parameters is performed as in Model A and Model B.

$$
\mathrm{H} 2=\frac{\mu_{N}(x)^{0.2} * \mu_{I}(x)^{1.1}}{\mu_{N}(x)^{0.2}+\mu_{I}(x)^{1.1}-\mu_{N}(x)^{0.2} * \mu_{I}(x)^{1.1}}
$$


where $\mu_{N}(x), \mu_{I}(x)$ are the membership values of NS1 and IgG respectively and NS1 is diluted by 0.2 and IgG is concentrated by 1.1 as in Model B.

The Hamacher results in (11) and (13) are then combined using the Ordered Weighted Aggregation Operator (OWA) as in (14).

$$
\mathrm{OWA}=0.4 * \operatorname{maximum}\{\mathrm{H} 1, \mathrm{H} 2\}+0.6 * \operatorname{minimum}\{\mathrm{H} 1, \mathrm{H} 2\}
$$

The weights of the OWA operator are determined from Model A.

The ambiguous region of the model is constructed using the concepts in Model A and Model B and the ambiguous region is found to lie in between the values 0.4413 and 0.5727 . Thus, values above 0.5727 indicate the region with DF patients (non-severe) whereas the region with DHF patients (severe) is shown for values less than 0.4413 .

When the model is validated at 96 hours from onset of illness, it could be seen that $58.8 \%$ of the DHF patients are correctly classified into severe region. Although this model has a much lower percentage of misclassifications than Model A and Model B, still the model misclassifies $41.2 \%$ of DHF patients into ambiguous and non-severe (DF) regions. This is a costly error as, by classifying a patient as DF who indeed should be classified as a DHF patient, the patient may not receive proper attention and thus the patient can easily proceed into critical stage without being detected.

Therefore, the existing model is further improved by using information from the decision trees evaluated at 108 hours from onset of illness (Fig.3 and Fig.4). Since, Fig.3 uses PAF and Fig.4 uses platelet count in the decision process, the model is further improved to include the effect from the parameters PAF and Platelet count. Since the model in previous stage misclassifies DHF patients into non-severe region, the patients with initial model operator values above 0.4413 are subjected to second decision making which includes PAF and Platelet count as given in equation (14).

$\mathrm{H} 3=\frac{\mu_{P}(x)^{4} * \mu_{P A}(x)^{4}}{\mu_{P}(x)^{4}+\mu_{P A}(x)^{4}-\mu_{P}(x)^{4} * \mu_{P A}(x)^{4}}$

where $\mu_{P}(x), \mu_{P A}(x)$ are the membership values of Platelet and PAF respectively and both are concentrated by 4 .

For these patients, after going through the second decision criteria, the final model output result is obtained through equation (15).

$\mathrm{OWA}_{\text {new }}=0.4 *$ maximum $\{\mathrm{OWA}, \mathrm{H} 3\}+0.6 * \operatorname{minimum}\{\mathrm{OWA}, \mathrm{H} 3\}$ (15)

When this improved model which includes second testing with PAF and platelet count is validated at 96 hours from onset of illness, it can be seen that the model output results have improved for the DHF patients than it was in the previous stage, as now $82.3 \%$ of DHF patients are correctly classified. Without testing for PAF and Platelet counts five DHF patients (29.4\%) were misclassified and two $(11.76 \%)$ were in the ambiguous region. However, after considering their PAF and platelet counts there were only two 
misclassifications of DHF patients and only one DHF patient fell into the ambiguous region.

At 96 hours from onset of illness, the final model performed with an accuracy of $76.19 \%$. This improved model, implemented using decision tree results performs with much higher accuracy than the individual models developed in Jayasundara, et al. (2016) and Premaratne, et al. (2017). The final validation results of the three approaches are shown in Table 1. From Table 1, it can be seen that the decision tree informed model performs the best with an accuracy of $76.19 \%$.

Table 1: Accuracy of the models evaluated at 96 hours from onset of illness.

\begin{tabular}{|c|c|c|c|c|}
\hline Model A & Model B & $\begin{array}{c}\text { Model in 1 } \\
\text { approach }\end{array}$ & $\begin{array}{c}\text { Model in } 2^{\text {nd }} \\
\text { approach }\end{array}$ & $\begin{array}{c}\text { Model in } 3^{\text {rd }} \\
\text { approach }\end{array}$ \\
\hline $71.43 \%$ & $53.00 \%$ & $58.82 \%$ & $64.7 \%$ & $76.19 \%$ \\
\hline
\end{tabular}

As the decision tree informed model ( $3^{\text {rd }}$ approach) performed with an accuracy of $76.19 \%$ at 96 hours from onset of illness, and resulted in $82.3 \%$ of correct DHF classification, this model can be used for early decision making. This model uses a reduced number of parameters than the previous studies Jayasundara, et al. (2016) and Premaratne, et al. (2017). The cytokines IL-1 $\beta$ and S1P and the immune parameter lymphocyte count are not used in this model. This makes it a relatively cost effective model as well. The accuracy of this model at 96 hours from onset of illness is highly improved than Models A and B. This indicates that the model with combined interactions between inflammatory mediators and immune parameters performs well than when they are used individually to measure dengue severity and that decision trees play a vital role in making decisions about parameter selection for the decision model.

\section{Sensitivity analysis}

Sensitivity analysis is performed to see how the decision tree based model outcomes would be affected when the degrees of fuzziness are changed. In our model, we have our ambiguous region between 0.4413 and 0.5727. Patients that show model values below 0.4413 are categorized as DHF and, those who show values above 0.5727 are categorized as DF. Therefore, our classification as to DF or DHF is based on the upper and lower boundaries of the ambiguous region. Thus, it is important to see how sensitive these boundary values are and if their change can affect in patients' classification. Fig. 5 denotes the change in the ambiguous region when the lower and upper boundary of the membership functions are changed over a small range and Fig.6 denotes the change in the ambiguous region when the amounts of concentration and dilution are changed. 
Model output result is based on the membership value, and the corresponding membership value depends on the chosen cut off values used to develop the membership function. There's always variability and imprecision associated with these cut off values as, variability and imprecision are inherently associated with cytokine and immune parameter levels measured in dengue patients. When the membership cut off points are changed by a small amount, if the resulting ambiguous region vary vastly, then the patient's classification will not be consistent and the model becomes less robust. As, it can be seen from Fig.5, when the membership cut off values are changed, the ambiguous region does not vary in a way that can change the classification group of a patient.

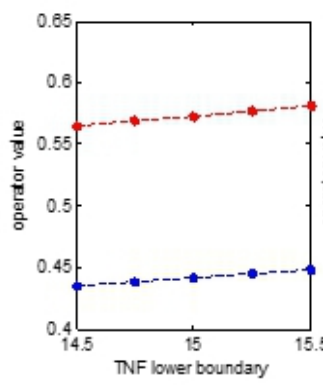

(a)

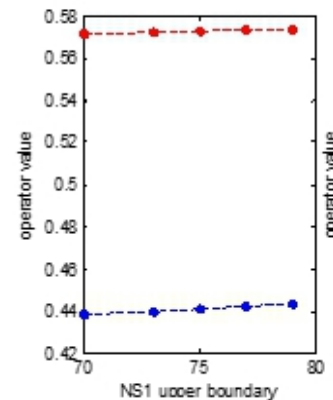

(d)

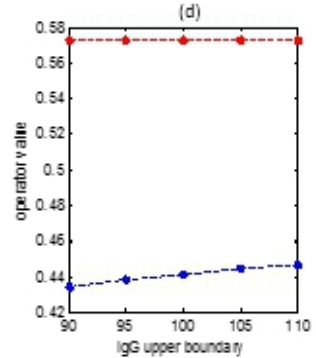

(g)

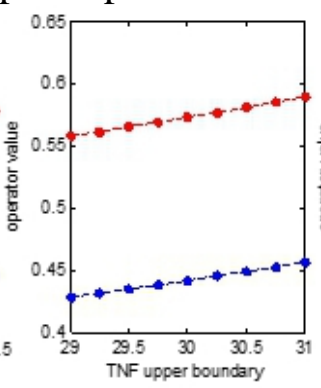

(b)
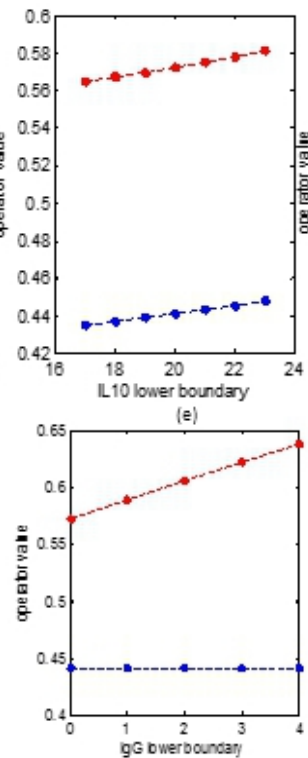

(h)

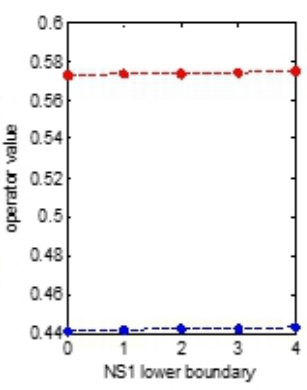

(c)

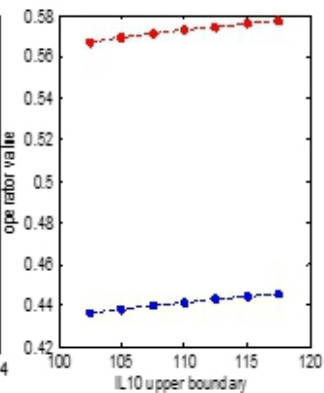

(f)

Fig.5: Behaviour of the ambiguous region when the lower cut off value and the upper cut off value of the membership function is changed for the parameters TNF (a, b), NS1 (c, d), IL$10(\mathrm{e}, \mathrm{f}), \operatorname{IgG}(\mathrm{g}, \mathrm{h})$. 
Concentration and dilution are performed to intensify the impact from the more important variables. These weights are chosen in a way that they give prominence to these parameters and at the same time would not make the model to be biased towards DHF patients. It is important to see how the model results would be affected if these concentration weights are chosen differently. As, it can be seen from Fig.6, when different weights are chosen, the ambiguous region does not vary in a way that can alter the classification group of a patient, as the ambiguous boundary levels do not deviate far from our original levels of 0.4413 and 0.5727 even when the concentration and dilution weights are changed.

Sensitivity analysis is especially important when working with a small limited sample, such as ours, as a small change in the patient decision can hugely affect the overall model performance and accuracy. Both Fig.5 and Fig. 6 indicate that the model is robust.

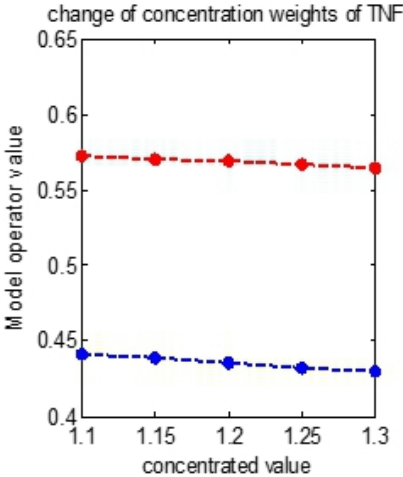

(a)

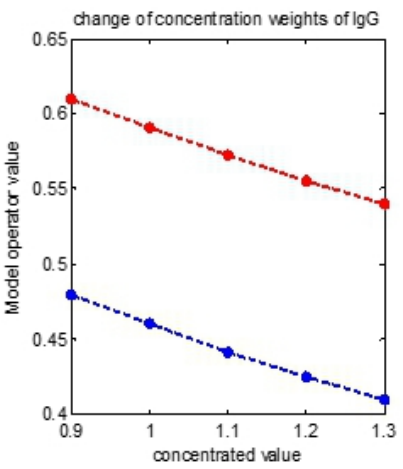

(c)

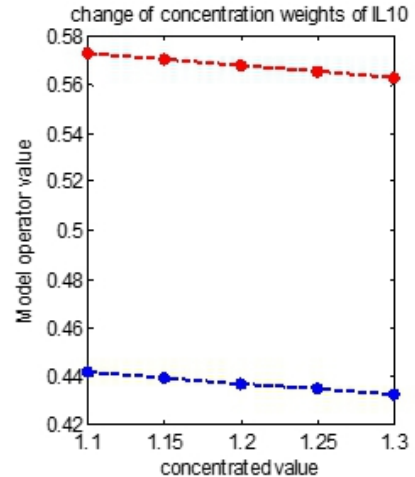

(b)

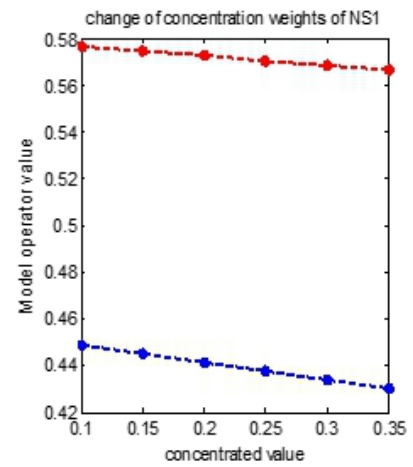

(d)

Fig.6: Behaviour of ambiguous region for a change in concentration weights for TNF (a), IL-10 (b), IgG (c), NS1 (d). 


\section{Conclusion}

This study is an attempt to develop a decision model to predict the severity level of dengue patients and is an improved extension of the models in (Jayasundara, et al., 2017) and (Premaratne, et al., 2017). Three variations are carried out. In the decision tree based model, IID3 decision trees are used to determine which inflammatory mediators and immune parameters should be used in constructing the decision model.

It could be seen that the decision tree based model, performs with the highest accuracy and that the other two models succumb to the more serious error of misclassifying DHF patients in to the non-severe region.

The decision tree based model makes decisions mainly based on the parameters NS1, IgG, TNF- $\alpha$ and IL-10. Second decision criteria is made using PAF and platelet counts. Unlike in Jayasundara, et al. 2016 this model uses only three inflammatory mediators for the decision making and this can be economically beneficial. This model can be considered as an improved decision maker than the individual models in Jayasundara, et al. (2016) and Premaratne, et al. (2017). Although the model tends to overestimate the risk of DF patients, this model reduces the more serious risk of incorrectly classifying DHF patients as DF patients. The model validation results for patients at 96 hours from onset of illness achieved an accuracy of $76.19 \%$ and only 2 DHF patients (out of 17) were misclassified. The sensitivity analysis indicated the model to be robust. When working with a small data set a slight change in the decision can hugely affect the overall performance of the model. Thus, sensitivity analysis is useful to generalize the model as it is based on a limited data set. Therefore, this model will be useful as an early indicator to detect dengue severity.

The drawback of this model is it tend to overestimate the risk of DF patients. In the final decision tree informed model 3 out of 4 DF patients were misclassified into severe region. Also, all the patients considered in this sample are adult patients and it is important to test the decision rules on samples which include children as well, as severe dengue and death is common among children (World Health Organization, 2016).

\section{References:}

1. Adeli, A., \& Neshat, M. (2010). A Fuzzy Expert System for Heart Disease Diagnosis . Proceedings of the Internatonal MultiConference of Engineering and Computer Scientists. HongKong.

2. Anand, S. K., Kalpana, R., \& Vijayalakshmi, S. (2013). Design and Implementation of a Fuzzy expert system for detecting and estimating the level of asthma and chronic obstructive pulmonary disease. World Applied Sciences Journal, 213-223. 
3. Appanna, R., Wang, S. M., Ponnampalavanar, S. A., LumL.C.S., \& Sekaran, S. D. (2012). Cytokine factors present in dengue patient sera induces alterations of junctional proteins in human endothelial cells. Am J Trop Med Hyg, 87, 936-942.

4. Bozza, F. A., Cruz, O. G., Zagne, S. M., Azeredo, E. L., \& Nogueira, R. M. (2008). Multiplex cytokine profile from dengue patients: MIP1 beta and IFN-gamma as predictive factors for severity. $B M C$ Infectious Diseases, 8(86).

5. Brasier, A. R., Ju, H., Garcia, J., Spratt, H. M., Victor, S. S., \& Forshey, B. M. (2012). A three component biomarker panel for prediction of dengue hemorrhagic fever. Am J Trop Med, 86(2), 341348.

6. Chen, L., Lei, H., Liu,C., Shiesh, S., Chen, S., \& Liu,H.,...Yeh,T. (2006). Correlation of serum levels of macrophage migration inhibitory factor with disease severity and clinical outcome in dengue patients. Am J Trop Med Hyg. , 74, 142-147.

7. de Azeredo, E. L., Monteiro, R. Q., \& de-Oliveira Pinto, L. M. (2015). Thrombocytopenia in Dengue: Interrelationship between Virus and the Imbalance between Coagulation and Fibrinolysis and Inflammatory Mediators. Mediators of Inflammation, 2015.

8. Gomes, L., Fernando, S., Fernando, R. H., Wickramasinghe, N., Shyamali, N. L., \& Ogg, G. S. (2014). Sphingosine 1-Phosphate in Acute Dengue Infection. PloS ONE, 9(11).

9. Green, S., Vaughan, D., Kalayanarooj, S., Nimmannitya, S., Suntayakorn, S., Nisalak, A., \& Ennis, F. (1999). Elevated plasma interleukin-10 levels in acute dengue correlate with disease severity. J Med Virol, 59, 329-334.

10. Jayasundara, S.D.P., Perera, S. S.N., Malavige, G. N., \& Jayasinghe, S. (2017). Mathematical modelling and a systems science approach to describe the role of cytokines in the evolution of severe dengue. $B M C$ Systems Biology, 11(34).

11. Jin, C., De-lin, L., \& Fen-xiang, M. (2009). An Improved ID3 Decision Tree Algorithm. International Conference on Computer Science and Education.

12. Kalpana, M., \& Senthil, K. A. (2011). Fuzzy Expert system for diabetes using Fuzzy Verdict Mechanism. Int J Advanced Networking and Apllications, 1128-1134.

13. Kittugul, L., Temprom, W., Sujirara, D., \& Kittugul, C. (2000). Determination of tumor necrosis factor- alpha in dengue virus infected patients by sensitive biotin-streptravidin enzyme-linked immunosorbent assay. J Virol Methods., 90, 51-57. 
14. Malavige, G. N., Gomes, L., Alles, L., Chang, T., Salimi, M., Fernando, S.,... Ogg,G.S. (2013). Serum IL-10 as a marker of severe dengue infection. BMC Infect Dis., 13(341).

15. Neshat, M., Yaghobi, M., Naghibi, M. B., \& Esmaelzadeh, A. (2008). Fuzzy Expert system design for diagnosis of liver disorders.

16. Paranavitane, S. A., Gomes, L., Kamaladasa, A., Adikari, T. N., Wickramasinghe, N., Jeewandara, C., . . . Malavige, G. N. (2014). Dengue NS1 antigen as a marker of severe clinical disease. $B M C$ infectious diseases, 14(570).

17. Perez, A., García, G., Sierra, B., Alvarez, M., Vázquez, S., \& Cabrera, M. (2004). IL-10 levels in Dengue patients: Some findings from the exceptional epidemiological conditions in Cuba. J Med Virol, 59, 230-234.

18. Potts, J. A., Gibbons, R. V., Rothman, A. L., Srikiatkhachorn, A., Thomas, S. J., \& Supradish, P.,...Kalayanarooj,S. (2010). Prediction of Dengue Disease Severity among Pediatric Thai patients using early clinical laboratory indicators. PLoS Negl Trop Dis, 4(8).

19. Premaratne, M. K., Perera, S. S.N., Malavige, G. N., \& Jayasinghe, S. (2017). Mathematical Modelling of Immune Parameters in the Evolution of Severe Dengue. Computational and Mathematical Methods in Medicine, 2017.

20. Stanaway, J. D., Shepard, D. S., Undurraga, E. A., Halasa, Y. A., Coffeng, L. E., \& Brady, O. J. (2016). The global burden of dengue: an analysis from the Global Burden of Disease Study 2013. Lancet Infect Dis., 16(6), 712-723.

21. World Health Organization. (2009). Dengue Guidelines for Diagnosis, Treatment, Prevention and Control. France.

22. World Health Organization. (2016, September 1). Dengue and Severe Dengue. Retrieved from World Health Organization: http://www.who.int/mediacentre/factsheets/fs117/en/

23. World Health Organization. (2016). Weekly Epidemiological Record. Geneva: World Health Organization.

24. Yager, R. R. (1988). On ordered weigted averaging aggregation operators in multicriteria decision making. IEEE Transactions on Systems, Man, and Cybernetics, 18, 183-190.

25. Yager, R. R. (1993). Families of OWA operators. Fuzzy Set Syst, 59, 125-48.

26. Zadeh, L. A. ( 1983). A computational approach to fuzzy quantifiers in natural languages. Comput Math Appl., 9, 149-184.

27. Zadeh, L. A. (1965). Fuzzy Sets. Information and Control, 8, 338353. 